\title{
First Results on Dark Matter Annual Modulation from the ANAIS-112 Experiment
}

\author{
J. Amaré, ${ }^{1,2}$ S. Cebrián, ${ }^{1,2}$ I. Coarasa, ${ }^{1,2}$ C. Cuesta, ${ }^{1, \$}$ E. García, ${ }^{1,2}$ M. Martínez, ${ }^{1,2,3}$ M. A. Oliván, ${ }^{1, \S}$ \\ Y. Ortigoza, ${ }^{1,2}$ A. Ortiz de Solórzano, ${ }^{1,2}$ J. Puimedón, ${ }^{1,2}$ A. Salinas, ${ }^{1,2}$ M. L. Sarsa, ${ }^{1,2, \uparrow}$ \\ P. Villar, ${ }^{1,2}$ and J. A. Villar ${ }^{1,2, *}$ \\ ${ }^{1}$ Laboratorio de Física Nuclear y Astropartículas, Universidad de Zaragoza, C/ Pedro Cerbuna 12, 50009 Zaragoza, Spain \\ ${ }^{2}$ Laboratorio Subterráneo de Canfranc, Paseo de los Ayerbe s.n., 22880 Canfranc Estación, Huesca, Spain \\ ${ }^{3}$ Fundación ARAID, Av. de Ranillas 1D, 50018 Zaragoza, Spain
}

(Received 12 March 2019; published 16 July 2019)

\begin{abstract}
ANAIS is a direct detection dark matter experiment aiming at the testing of the DAMA/LIBRA annual modulation result, which, for about two decades, has neither been confirmed nor ruled out by any other experiment in a model independent way. ANAIS - 112, consisting of $112.5 \mathrm{~kg}$ of sodium iodide crystals, has been taking data at the Canfranc Underground Laboratory, Spain, since August 2017. This Letter presents the annual modulation analysis of 1.5 years of data, amounting to $157.55 \mathrm{~kg} \mathrm{yr}$. We focus on the model independent analysis searching for modulation and the validation of our sensitivity prospects. ANAIS - 112 data are consistent with the null hypothesis ( $p$ values of 0.67 and 0.18 for [2-6] and [1-6] keV energy regions, respectively). The best fits for the modulation hypothesis are consistent with the absence of modulation $\left(S_{m}=-0.0044 \pm 0.0058 \mathrm{cpd} / \mathrm{kg} / \mathrm{keV}\right.$ and $-0.0015 \pm 0.0063 \mathrm{cpd} / \mathrm{kg} / \mathrm{keV}$, respectively). They are in agreement with our estimated sensitivity for the accumulated exposure, which supports our projected goal of reaching a $3 \sigma$ sensitivity to the DAMA/LIBRA result in five years of data taking.
\end{abstract}

DOI: 10.1103/PhysRevLett.123.031301

There is overwhelming evidence from cosmological and astrophysical observations supporting that a large fraction of the Universe energy-mass budget is not explained in the framework of the standard model of particle physics, assuming the cosmological standard model [1]. The solution to the dark matter (DM) and dark energy (DE) puzzle is probably of a complex nature. In one of the preferred hypothetical scenarios, DM can be understood as a new non-zero-mass particle having a very low interaction probability with baryonic matter. Although proposed candidates span about 45 orders of magnitude in mass, and 60 in cross section, axions and weakly interacting massive particles (WIMPs) are among the better motivated [2]. Experimental effort devoted to unraveling the nature of the DM particles has been spent, either by direct [3], indirect [4], or accelerator searches [5], which are complementary to each other. Only one experiment, DAMA/LIBRA [6-8], has provided a longstanding positive result: the observation of a highly statistically significant annual modulation in the detection rate, which is compatible with that expected for galactic halo dark matter particles. This result has neither been reproduced by any other experiment, nor ruled out in a model independent way. Compatibility among the different experimental results in most conventional WIMP-DM scenarios is actually disfavored [9-19]. Then, a similar annual modulation search using the same target is mandatory to shed light on the DAMA/LIBRA conundrum, which is the goal of the ANAIS (annual modulation with
$\mathrm{NaI}$ scintillators) experiment. Other efforts sharing the ANAIS goal in the international dark matter community are the COSINE-100 experiment, which also takes data in the dark matter mode at the Yang-Yang Underground Laboratory, South Korea [9,20,21], and, in the longer term, SABRE, which aims at installing twin detectors in Australia and Italy [22], and COSINUS, which is developing cryogenic detectors based on NaI [23].

An annual modulation in the dark matter interaction rate is expected by the revolution of Earth around the Sun, which distorts the DM particle velocity distribution function as seen by the detector, which is typically assumed to be Maxwellian boosted by the Sun velocity [24-26]. The effect is present unless the DM halo is corotating with the Solar System. However, it is strongly dependent on the specific halo model both in amplitude and in phase. It is natural to assume that the Sun is moving through a locally isotropic DM halo, with the Earth orbiting aside. Consequently, searches are performed for a modulation of DM-like events with a one year period and a welldefined phase. On the other hand, preferably, an annual modulation analysis should not assume a priori neither the period of the modulation nor the phase, but it should derive them from the data. A full and consistent analysis, then, requires several years of measurement in very stable conditions. This is the long-term goal of our experiment.

ANAIS - 112, consisting of $112.5 \mathrm{~kg}$ of $\mathrm{NaI}(\mathrm{Tl})$ detectors, was installed in 2017 at the Canfranc Underground 
Laboratory, LSC, in Spain. The ANAIS - 112 setup undergoes a different residual cosmic ray flux and environmental conditions than DAMA/LIBRA ( $800 \mathrm{~m}$ versus $1400 \mathrm{~m}$ rock overburden, for instance). Consequently, the potential confirmation of a modulation with same phase and amplitude would be very difficult to explain as an effect of backgrounds or systematics. ANAIS - 112 experimental details have been recently reported, as well as the performance of the first year's operation [27], the analysis of backgrounds [28], and the sensitivity prospects for a fiveyear operation [29]; here we just briefly summarize the most relevant features of the experimental apparatus.

ANAIS - 112 uses nine $\mathrm{NaI}(\mathrm{Tl})$ modules produced by Alpha Spectra Inc. in Colorado (US) [30]. These modules have been manufactured from 2012 to 2017 and shipped to Spain, avoiding air travel in order to prevent the cosmogenic activation of the module materials. Each crystal is cylindrical (4.75" diameter and 11.75 " length), with a mass of $12.5 \mathrm{~kg}$, and it is housed in OFE (oxygen free electronic) copper. This encapsulation has a Mylar window, which allows for a low energy calibration using external gamma sources. It incorporates two quartz optical windows to couple the photomultipliers (PMTs). All PMT units, as well as all relevant materials used in the building of the detectors, have been screened for radiopurity using high purity germanium detectors in the low background facilities at LSC. Their contribution to the experiment background has been estimated $[28,31]$ and included in our background model (see below). We would like to emphasize that the outstanding light collection measured for the nine modules is at the level of 15 photoelectrons (phe) per keV [27,32]. ANAIS - 112 is calibrated every two weeks using external ${ }^{109} \mathrm{Cd}$ sources: all the nine modules are simultaneously calibrated using a multisource system that minimizes down time periods. Background events from the decay of ${ }^{40} \mathrm{~K}$ and ${ }^{22} \mathrm{Na}$ in the crystal bulk, associated to 3.2 and $0.9 \mathrm{keV}$ energy depositions, and selected by coincidence with an energy deposition in a second module around 1461 and $1275 \mathrm{keV}$, respectively, are also used to improve the accuracy of the calibration down to the energy threshold [27].

The ANAIS - 112 shielding consists of $10 \mathrm{~cm}$ of archaeological lead, $20 \mathrm{~cm}$ of low activity lead, an antiradon box (continuously flushed with radon-free nitrogen gas), an active muon veto system made up of 16 plastic scintillators designed to cover the top and sides of the whole ANAIS setup, and $40 \mathrm{~cm}$ of neutron moderator (a combination of water tanks and polyethylene blocks). In the design of the muon veto system we follow a tagging strategy instead of a hardware vetoing. The goal is twofold: on the one hand, to discard events in the $\mathrm{NaI}(\mathrm{Tl})$ crystals coincident with muon veto triggers; on the other hand, to analyze eventual correlations between muon hits in the plastic scintillators and events in the $\mathrm{NaI}(\mathrm{Tl})$ crystals, especially in the region of interest (ROI) [1-6] keV (energy will be shown in electron equivalent units throughout this
Letter). Environmental parameters (muon flux, radon content in the laboratory air, temperature at the detectors and electronics, etc.) are continuously monitored [27,33].

The ANAIS - 112 electronic chain and data acquisition system (DAQ) have been described in Refs. [27,33]. Each PMT charge signal is independently processed and divided into (1) a trigger signal, (2) a low energy (LE) signal that goes to the digitizers which sample the waveforms at 2 Gs/s with high resolution (14 bits), and (3) a high energy (HE) signal, conveniently attenuated. The trigger of each PMT signal is done at phe level, while the single module trigger is done by the coincidence (logical AND) of the two PMT triggers in a $200 \mathrm{~ns}$ window. The global trigger is the logical OR of the nine modules' trigger signals. Trigger efficiency is close to $100 \%$ down to the analysis threshold established at $1 \mathrm{keV}$ [27].

ANAIS - 112 started taking data in the DM mode on August 3, 2017. It has accumulated almost 1.5 years of data-taking time in quite stable conditions by February 12, 2019. Total live time available for the annual modulation analysis is 527.08 days: 341.72 (first year) +185.36 (half second year). This implies a live time of $94.5 \%$ $(95.2 \%)$, dead time of $2.9 \%(2.2 \%)$, and down time of $2.6 \%(2.6 \%)$ for the first (second) year of data taking, respectively. The down time is mainly due to the periodical calibration runs carried out using low energy gamma sources. We remove events arriving less than one second after the last muon veto trigger, correcting the total live time by subtracting one second per muon veto trigger, so the live time used for the annual modulation analysis that follows is 511.16 days.

A blind analysis strategy in three levels has been followed: first, we calculate pulse parameters, the time since the last muon veto, and we apply the peak-finding algorithm to identify individual phe in low energy pulses; second, we calibrate the energy response of every detector at LE and HE [27]; third, we optimize the pulse shape cuts and calculate their efficiency, being the LE variable hidden for events corresponding to single hits ( $M 1$ events). Only $10 \%$ of the data were unblinded for the general background assessment and the fine tuning of procedures [27]; those data were randomly chosen (34 days amounting to 32.9 days live time) and time evolution was kept hidden until the data unblinding, presented in this Letter.

Calibration procedures and efficiency corrections applied in the following have been derived as done in Ref. [27] for the first year, continuing with same procedure in the half second year added in this analysis. Events in the ROI are selected, after energy calibration, by imposing the following criteria: single hit events $(M 1)$; a pulse shape cut combining the fraction of the pulse area in [100-600] ns after the event trigger, defined following Ref. [34], and the logarithm of the mean time of the distribution of the individual phe arrival times in the digitized window [20]; and events having an asymmetric light sharing between the 
two PMT signals are removed by imposing a cut on the number of peaks identified in each PMT (asymmetry cut). The total detection efficiency, $\varepsilon(E, d)$, calculated independently for every detector $d$ as a function of the energy, $E$, can be written [27] as

$$
\varepsilon(E, d)=\varepsilon_{t r g}(E, d) \times \varepsilon_{P S A}(E, d) \times \varepsilon_{a s y}(E, d),
$$

where the trigger efficiency $\varepsilon_{t r g}(E, d)$ is calculated from Monte Carlo (MC) simulations, and the efficiencies of the pulse shape, $\varepsilon_{P S A}(E, d)$, and asymmetry, $\varepsilon_{a s y}(E, d)$, cuts are evaluated from the 3.2 and $0.9 \mathrm{keV}$ events selected by the coincidence with the high energy gammas following ${ }^{40} \mathrm{~K}$ and ${ }^{22} \mathrm{Na}$ decays, and ${ }^{109} \mathrm{Cd}$ calibration events, respectively, accumulated for all the analyzed exposure. Total detection efficiency ranges from 0.15 to 0.35 at $1 \mathrm{keV}$, depending on the detector, increases up to 0.8 at $2 \mathrm{keV}$, and is nearly 1 at $4 \mathrm{keV}$ for all the modules. Statistical errors in the total efficiency vary from 3 to $8 \%$ at $1 \mathrm{keV}$ down to $1 \%$ at $6 \mathrm{keV}$. Comparing different methods for the efficiency calculation we have also estimated a systematic uncertainty that amounts up to $20 \%$ at $1-1.2 \mathrm{keV}$ and is negligible above $1.5 \mathrm{keV}$. More details can be found in Ref. [27]. Time evolution of these efficiencies is under study.

The background model for all of the nine detectors used in the ANAIS - 112 setup has been developed. It is based on $\mathrm{MC}$ simulations using the measured activity in external components and in crystals, including cosmogenic products, quantified in dedicated, independent measurements using different analysis techniques. It provides a good overall description of measured data at all energy ranges above $2 \mathrm{keV}$ and at different analysis conditions (coincidence or anticoincidence) [28]. In the ROI the background is dominated by the emissions from the crystals themselves, in particular, ${ }^{210} \mathrm{~Pb}(32.5 \%)$ and ${ }^{3} \mathrm{H}(26.5 \%)$ continua, and ${ }^{40} \mathrm{~K}(12 \%)$ and ${ }^{22} \mathrm{Na}(2.0 \%)$ peaks are the most significant contributions. Short-lived isotopes activated cosmogenically are still present in the bulk of the last received crystals $[35,36]$, contributing as background in the ROI, specially in the [3-5] keV region. However, from 1 to $2 \mathrm{keV}$ there is a large fraction of our background lacking from explanation in the frame of the ANAIS-112 background model, as stated in Ref. [28]. It could have as its origin a nonbulk scintillation leaking through our event selection criteria.

The time evolution of the rate of those events surviving all the cuts during the first year and a half of ANAIS - 112 operation is shown for different populations in Figs. 1(a)1(f). Data from all the modules have been added together and corrected by the corresponding efficiencies. The two lower panels correspond to two different energy windows in the ROI: [1-6] keV, (a), and [3-5] keV, (b), while the upper panels show the evolution of control populations for which no modulation is expected: (c) [6-20] keV, (d) double-coincidence events (M2) in energy region [1-6] keV, and coincident events attributed to (e) ${ }^{40} \mathrm{~K}$, and (f) ${ }^{22} \mathrm{Na}$

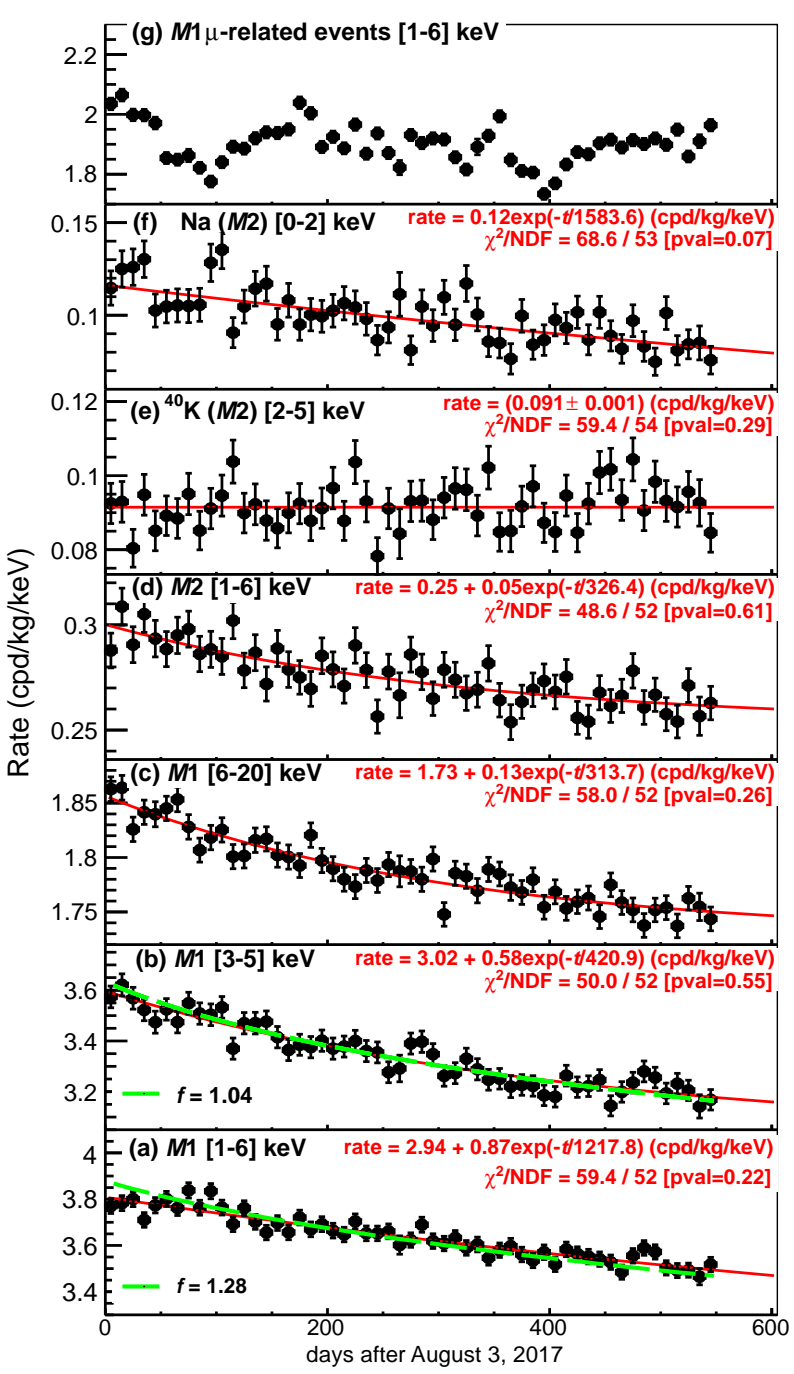

FIG. 1. Rate of events corresponding to different populations along the first year and a half of ANAIS - 112 operation, calculated in ten-day binning. Data from all the modules have been added together and corrected, when necessary, by the corresponding efficiencies. Events surviving all the filters are shown in panels (a)-(f): in energy region [1-6] $\mathrm{keV}$, (a); [3-5] keV, (b); [6-20] keV, (c); $M 2$ events in energy region [1-6] $\mathrm{keV},(\mathrm{d}) ; M 2$ events in energy region [2-5] $\mathrm{keV}$ in coincidence with a high energy gamma in a second detector in the ${ }^{40} \mathrm{~K}$ window, (e); and $M 2$ events in energy region [0-2] keV in coincidence with a high energy gamma in a second detector in the ${ }^{22} \mathrm{Na}$ window, (f). Events arriving less than one second after a muon veto trigger before the cuts are shown in panel $(\mathrm{g})$. Fits are shown as red solid lines, and corresponding fit parameters and chi-squared and $p$ values are also given in the plot. Green dashed lines correspond to the background model, normalized according to a factor, $f$, which is also given in the plots.

decays in the crystals (low energy $M 2$ events have an energy deposition in a second detector in a window around the corresponding high energy gamma). The uppermost panel, $(\mathrm{g})$, presents the evolution of muon-related lowenergy events before the cuts (M1 events in [1-6] keV 
region arriving less than 1 second after a muon veto trigger). As pointed out in Ref. [27], many low energy events are registered after a muon passage through the detector. They likely originate in the long tail of the scintillation pulse produced by a large muon energy deposition, which is able to trigger many times the DAQ system and produce fake low-energy events. This population is clearly fluctuating in time and will be studied in a future work.

In Figs. 1(a) and 1(b), we observe a relevant decrease in the rate at the ROI, which amounts up to $8 \%$ in the analyzed period. It is caused by cosmogenically activated isotopes and is well reproduced qualitatively in all energy windows by our background model [28], shown in Fig. 1 in green dashed lines. It is normalized by a factor, $f$, to be more easily compared with the measured rates. Agreement in [3-5] keV region is also quantitatively very good, $f=1.04$, as the background time evolution in this region is dominated by short-lived cosmogenic isotopes. However, our background model underestimates the rate in the [1-6] $\mathrm{keV}$ region $(f=1.28)$, because from 1 to $2 \mathrm{keV}$ our background model does not reproduce the measurement [28], as commented on above.

We perform a least-squares fit on Figs. 1(a) to 1(d) to a constant term plus an exponential function in order to account for the mentioned background reduction and following our background model [28]. The $\chi^{2} / N D F$ and the values of the fitted parameters are shown in the figure. Good fits are obtained, with $p$ values larger than 0.20 in all cases. The ${ }^{40} \mathrm{~K}(3.2 \mathrm{keV})$ and ${ }^{22} \mathrm{Na}(0.9 \mathrm{keV}) \mathrm{M} 2$ populations, on the other hand, are modeled by a constant and an exponential decay, respectively. We derive consistent results ( $p$ value $=0.29$ ) in the first case, and a reasonable agreement with the ${ }^{22} \mathrm{Na}$ half-life $\left(T_{1 / 2}=3.01 \pm 0.36 \mathrm{y}\right.$, $p$ value $=0.07)$ in the second.

In this Letter we present our modulation results in two energy regions, [2-6] keV and [1-6] $\mathrm{keV}$, to allow for a direct comparison with the DAMA/LIBRA results. The values of the modulation amplitude observed by DAMA/ LIBRA, $S_{m}^{\text {DAMA }}$, are $0.0102 \pm 0.0008$ and $0.0105 \pm$ $0.0011 \mathrm{cpd} / \mathrm{kg} / \mathrm{keV}$ in the full exposure for [2-6] keV and using only phase 2 data for [1-6] keV energy region, respectively [8]. We expect results derived from [2-6] keV to be more robust because our data selection efficiencies strongly go down below $2 \mathrm{keV}$, increasing the risk to be affected by unknown systematics.

We evaluate the statistical significance of a possible modulation in our data by a least-squares method in the time-binned data. We have considered mean efficiencies for correcting the events rate at each bin, which implies there are correlation terms. However, being the uncertainty at each bin dominated by statistics, the effect of this correlation in the result should be small, and it has not been considered. The efficiency-corrected rate of events surviving the cuts in the [1-6] and [2-6] keV energy regions is modeled as

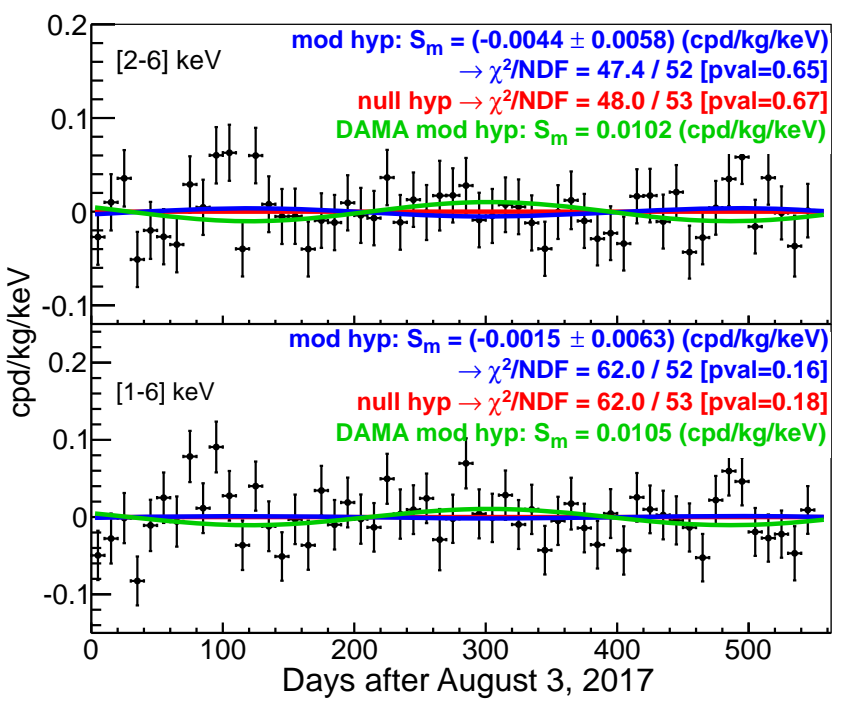

FIG. 2. ANAIS - 112 data in the energy windows [1-6] keV (bottom panel) and [2-6] keV (top panel) surviving all the cuts and efficiency corrected [27]. Data are displayed after subtracting the constant and exponential functions fitted to Eq. (2). Fits are also shown in the same way, both in the modulation (three free parameters) and the null hypothesis (two free parameters). $\chi^{2}$ and $p$ values displayed allow the comparison of both hypothesis, and DAMA/LIBRA results on modulation amplitude in both energy windows are shown in green [8].

$$
R(t)=R_{0}+R_{1} \exp (-t / \tau)+S_{m} \cos [\omega(t+\phi)],
$$

where $R_{0}$ and $R_{1}$ are free parameters and $\tau$ is fixed to the value obtained from our background model in the corresponding energy range. We also fix the period $(\omega=2 \pi /$ $\left.365 \mathrm{~d}=0.01721 \mathrm{rad} \mathrm{d}^{-1}\right)$ and the phase $(\phi=+62.2 \mathrm{~d}$, corresponding the cosine maximum to June 2 when taking as time origin August 3), while $S_{m}$ is fixed to 0 for the null hypothesis and left unconstrained (positive or negative) for the modulation hypothesis. This allows a direct comparison with the results from the DAMA/LIBRA analysis with one free parameter [8]. We present the best fit for both hypothesis for a ten-day time binning in Fig. 2. In order to highlight the presence or absence of modulation, we plot the data with the constant and exponential terms subtracted. For the sake of comparison, in the plot, we show the modulation measured by DAMA/LIBRA (green lines).

In both energy regions the null hypothesis is well supported by the $\chi^{2}$ test, with $\chi^{2} / N D F=48.0 / 53$ for the [2-6] $\mathrm{keV}(p$ value $=0.67)$ and $\chi^{2} / N D F=62.0 / 53$ for the [1-6] $\mathrm{keV}$ regions ( $p$ value $=0.18$ ). The best fits for the modulation hypothesis are $S_{m}=-0.0044 \pm$ $0.0058 \mathrm{cpd} / \mathrm{kg} / \mathrm{keV}$ and $-0.0015 \pm 0.0063 \mathrm{cpd} / \mathrm{kg} / \mathrm{keV}$ for [2-6] $\mathrm{keV}$ and [1-6] keV, respectively. In both cases, $p$ values are slightly lower than those of the null hypothesis ( 0.65 and 0.16 , respectively). The best fits are incompatible at $2.5 \sigma(1.9 \sigma)$ with the DAMA/LIBRA signal.

The statistical significance of our result is determined by the standard deviation of the modulation amplitude 


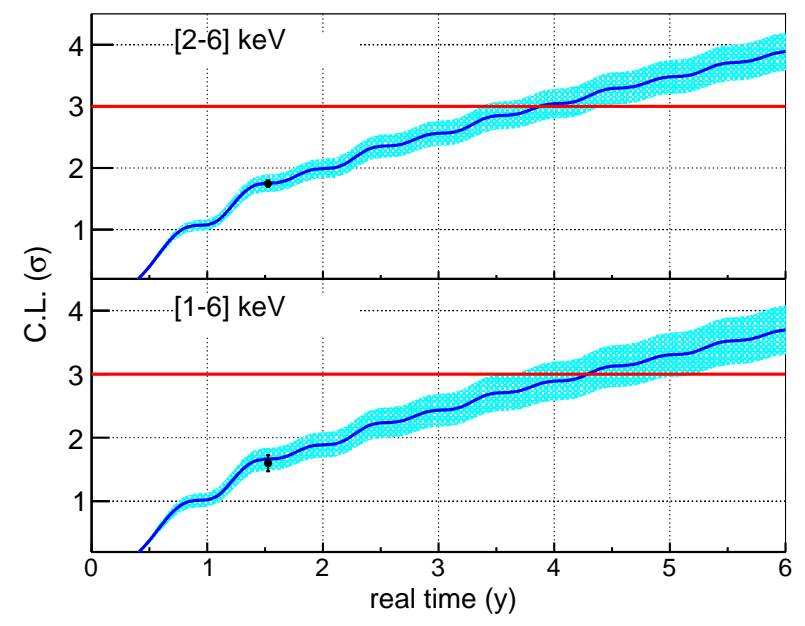

FIG. 3. ANAIS - 112 sensitivity to the DAMA/LIBRA signal in $\sigma$ C.L. units (see text) as a function of real time in the [2-6] keV (upper panel) and [1-6] keV (lower panel) energy regions. The black dots are the sensitivities derived in this work, $\sigma\left(S_{m}\right)$. The blue bands represent the $68 \%$ C.L. DAMA/LIBRA uncertainty [8].

distribution, $\sigma\left(S_{m}\right)$, which would be obtained in a large number of experiments like ANAIS - 112 with the present exposure. Then, we quote our sensitivity to DAMA/LIBRA result as the ratio $S_{m}^{\mathrm{DAMA}} / \sigma\left(S_{m}\right)$, which directly gives in $\sigma$ units the C.L. at which we can test the DAMA/LIBRA signal. At present, our result $\sigma\left(S_{m}\right)=0.0058(0.0063) \mathrm{cpd} /$ $\mathrm{kg} / \mathrm{keV}$ for [2-6] $\mathrm{keV}$ ([1-6] $\mathrm{keV}$ ) corresponds to a sensitivity of $1.75 \sigma(1.66 \sigma)$ to the DAMA/LIBRA signal. In Ref. [29] we found an analytical expression to calculate $\sigma\left(S_{m}\right)$ at a given exposure from the measured background and detection efficiency. Figure 3 (dark blue lines) displays our sensitivity projection calculated following Ref. [29] for the two studied energy ranges, whereas the blue bands represent the $68 \%$ uncertainty in $S_{m}^{\text {DAMA }}$ as reported in Ref. [8]. In the calculation we take into account the ANAIS - 112 live time distribution, the background reduction expected due to decaying isotopes and the statistical error in the detection efficiency. The black dots are the sensitivities derived in this work, including a systematic error estimated by changing the time binning from 1 to 20 days, and considering the systematics in the efficiency [27]. The results perfectly agree with our estimates, confirming the ANAIS - 112 projected sensitivity to the DAMA/LIBRA result. A $3 \sigma$ sensitivity should be at reach in 4-5 years of data taking.

Finally, Fig. 4 presents the best fit amplitudes, $S_{m}$, calculated per $1 \mathrm{keV}$ energy bins, from 1 to $20 \mathrm{keV}$ (bottom panel, black dots), together with the DAMA-phase-2 modulation amplitudes extracted from Ref. [8] (blue triangles). The top panel shows the $p$ values for the null (open squares) and modulation hypothesis (closed circles) for every energy bin. All the modulation amplitudes in the ROI are compatible with 0 and, in general, $p$ values

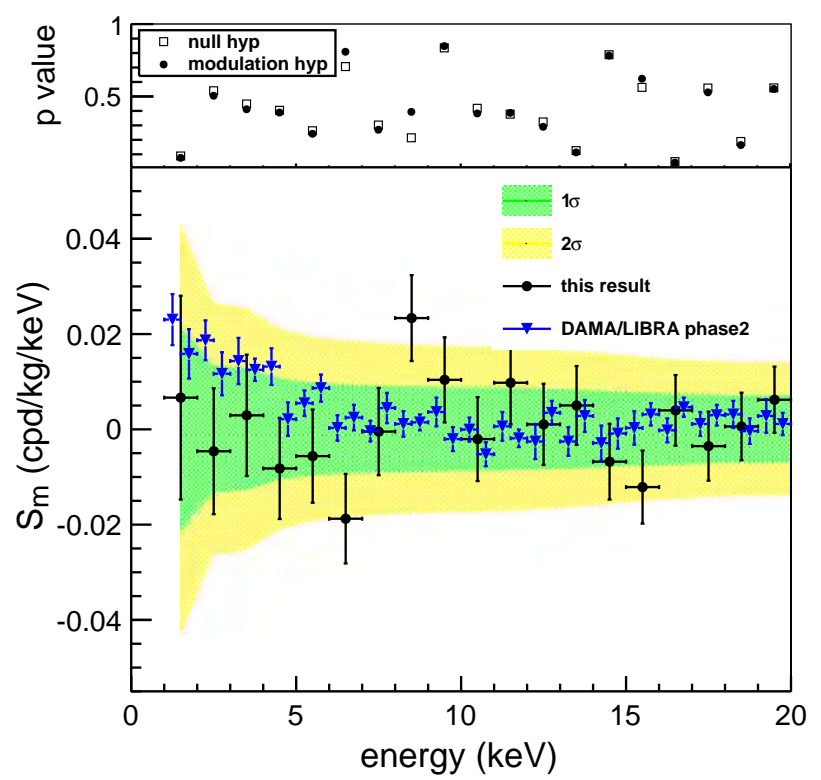

FIG. 4. Modulation amplitude per $1 \mathrm{keV}$ energy bins combining data from all the modules. We show for reference the corresponding DAMA/LIBRA result [8], and the $1 \sigma$ and $2 \sigma$ ANAIS 112 bands following the analysis done in Ref. [29]. Top panel compares the $p$ values of the fits to Eq. (2) with those corresponding to the null hypothesis for every energy bin.

for the null hypothesis are slightly larger than those of the modulation hypothesis. The $1 \sigma$ and $2 \sigma$ bands shown in the figure are obtained following Ref. [29] for the present ANAIS - 112 exposure.

In summary, to test the DAMA/LIBRA annual modulation result in a model independent way, an analysis of the first year and a half of data from ANAIS - 112 experiment has been presented. This analysis is based in visible energies (keVee), as DAMA/LIBRA does. This implies the interpretation in terms of dark matter is not direct, because it depends on some issues related with the detectors' performance. We are working on the measurement of the quenching factor for the scintillation of nuclear recoils in ANAIS-112 crystals, the most relevant parameter to allow such a comparison. Moreover, understanding nonbulk scintillation events populations is crucial: for instance, because of our high light yield, the contribution from noise events should appear in ANAIS at lower equivalent energies than in DAMA/LIBRA. Other experimental features, such as energy resolution, could have some impact in the comparison of results among experiments, but their effect should be minor. It will be analyzed in more depth in subsequent analysis. ANAIS - 112 annual modulation result is compatible with the sensitivity estimates done in Ref. [29], and confirms our goal of reaching sensitivity at $3 \sigma$ level in five years (see Fig. 3) to the DAMA/LIBRA result. We derive best fits for the modulation amplitude $S_{m}=-0.0044 \pm 0.0058$ and $-0.0015 \pm$ $0.0063 \mathrm{cpd} / \mathrm{kg} / \mathrm{keV}$, in the [2-6] and [1-6] keV energy 
regions, respectively, compatible with the absence of modulation.

This work has been financially supported by the Spanish Ministerio de Economía y Competitividad and the European Regional Development Fund (MINECO-FEDER) under Grants No. FPA2014-55986-P and No. FPA2017-83133-P, the Consolider-Ingenio Programme under Grants No. MultiDark CSD2009-00064 and No. CPAN CSD2007-00042, the LSC Consortium, and the Gobierno de Aragón and the European Social Fund (Group in Nuclear and Astroparticle Physics and I. Coarasa predoctoral grant). We thank the support of the Spanish Red Consolider MultiDark FPA2017-90566-REDC. We acknowledge the technical support from LSC and GIFNA staff.

Note added in proof.-Recently, we became aware of the COSINE-100 results on annual modulation [37].

*Deceased.

†mlsarsa@unizar.es

*Present Address: Centro de Investigaciones Energéticas, Medioambientales y Tecnológicas, CIEMAT, 28040, Madrid, Spain.

${ }^{\S}$ Present Address: Fundación CIRCE, 50018, Zaragoza, Spain.

[1] G. Bertone and D. Hooper, Rev. Mod. Phys. 90, 045002 (2018).

[2] L. Baudis, J. Phys. G 43, 044001 (2016).

[3] J. Liu, X. Chen, and X. Ji, Nat. Phys. 13, 212 (2017).

[4] J. Conrad and O. Reimer, Nat. Phys. 13, 224 (2017).

[5] O. Buchmueller, C. Doglioni, and L. T. Wang, Nat. Phys. 13, 217 (2017).

[6] R. Bernabei et al. (DAMA Collaboration), Eur. Phys. J. C 56, 333 (2008).

[7] R. Bernabei et al., Eur. Phys. J. C 73, 2648 (2013).

[8] R. Bernabei et al., Universe 4, 116 (2018); Nucl. Phys. At. Energy 19, 307 (2018).

[9] G. Adhikari et al., Nature (London) 564, 83 (2018).

[10] M. Kobayashi et al. (XMASS Collaboration), arXiv:1808 .06177.
[11] D. S. Akerib et al. (LUX Collaboration), Phys. Rev. D 98, 062005 (2018).

[12] K. Abe et al. (XMASS Collaboration), Phys. Rev. D 97, 102006 (2018).

[13] E. Aprile et al. (XENON Collaboration), Phys. Rev. Lett. 118, 101101 (2017).

[14] C. Savage, K. Freese, P. Gondolo, and D. Spolyar, J. Cosmol. Astropart. Phys. 09 (2009) 036.

[15] E. Aprile et al. (XENON100 Collaboration), Science 349, 851 (2015).

[16] J. Herrero-Garcia, J. Cosmol. Astropart. Phys. 09 (2015) 012.

[17] S. Baum, K. Freese, and C. Kelso, Phys. Lett. B 789, 262 (2019).

[18] S. Kang, S. Scopel, G. Tomar, and J.-H. Yoon, J. Cosmol. Astropart. Phys. 07 (2018) 016.

[19] J. Herrero-Garcia, A. Scaffidi, M. White, and A. G. Williams, Phys. Rev. D 98, 123007 (2018).

[20] K. W. Kim et al. (KIMS Collaboration), J. High Energy Phys. 03 (2019) 194.

[21] G. Adhikari et al., Eur. Phys. J. C 78, 107 (2018).

[22] C. Tomei (SABRE Collaboration), Nucl. Instrum. Methods A 845, 418 (2017).

[23] G. Angloher et al., Eur. Phys. J. C 76, 441 (2016).

[24] A. K. Drukier, K. Freese, and D. N. Spergel, Phys. Rev. D 33, 3495 (1986).

[25] K. Freese, J. A. Frieman, and A. Gould, Phys. Rev. D 37, 3388 (1988).

[26] K. Freese, M. Lisanti, and C. Savage, Rev. Mod. Phys. 85, 1561 (2013).

[27] J. Amaré et al., Eur. Phys. J. C 79, 228 (2019).

[28] J. Amaré et al., Eur. Phys. J. C 79, 412 (2019).

[29] I. Coarasa et al., Eur. Phys. J. C 79, 233 (2019).

[30] See http://www.alphaspectra.com/.

[31] J. Amaré et al., Eur. Phys. J. C 76, 429 (2016).

[32] M. A. Oliván et al., Astropart. Phys. 93, 86 (2017).

[33] M. Oliván, Ph. D. thesis, Universidad de Zaragoza, 2016.

[34] R. Bernabei et al., Nucl. Instrum. Methods Phys. Res., Sect. A 592, 297 (2008).

[35] P. Villar et al., Int. J. Mod. Phys. A 33, 1843006 (2018).

[36] J. Amaré et al., J. Cosmol. Astropart. Phys. 02 (2015) 046.

[37] G. Adhikari et al., following Letter, Phys. Rev. Lett. 123, 031302 (2019). 